\title{
Selección por mérito genético en Cavia porcellus para reproduc- ción en función al índice de selección
}

\author{
Selection by genetic merit in Cavia porcellus for reproduction by their \\ selection index
}

José Saucedo U. ${ }^{1,3}$, Hurley Quispe C. ${ }^{1}$, José Mantilla G. ${ }^{2}$

\section{Resumen}

\begin{abstract}
El estudio se desarrolló en el «Centro de Producción de Genética Superior (CENPROGEN-SUP)» en el distrito de Valle de Condebamba, región Cajamarca, Perú, con el objetivo de seleccionar cuyes machos para reproducción en función a su índice de selección. Fueron utilizados 80 cuyes machos del ecotipo Cajamarca de la Facultad de Ingeniería en Ciencias Pecuarias (FICP puros y cruzados) de la Universidad Nacional de Cajamarca, así como del Valle de Mangallana y del Valle de Cholocal. Los cuyes fueron alimentados con alfalfa, concentrado y agua a voluntad durante 56 días. Se evaluó el incremento de peso diario (IPD) y total (IPT), peso final (PF), longitud corporal (LC) y conversión alimenticia (CA). Los FICP cruzados tuvieron valores significativamente mejores para IPD, IPT, PF y LC (17.6 $\pm 1.8 \mathrm{~g}, 979 \pm 98 \mathrm{~g}, 1328 \pm 134 \mathrm{~g}$ y $36.6 \pm 1.3 \mathrm{~cm}$, respectivamente) $(\mathrm{p}<0.05)$. Por otro lado, no hubo diferencias entre grupos para conversión alimenticia. Se encontró correlación alta y significativa entre PF con LC y consumo de alimento $(\mathrm{p}<0.05)$, y correlación inversa entre el IPT y CA $(\mathrm{p}<0.05)$. El máximo valor de índice de selección corresponde a los cuyes FICP cruzados (0.873), seguido por FICP puros (0.544), Valle de Mangallana (0.400) y Valle de Cholocal (0.346); asimismo, el 45\% del primer cuartil corresponde a FICP cruzados. Se concluye que el mejor germoplasma con superioridad genética se encuentra en la población de cuyes FICP cruzados.
\end{abstract}

Palabras clave: cuy; parámetros productivos; mérito genético; ecotipo Cajamarca; índice de selección

\section{AbSTRaCT}

The study was carried out in the «Centro de Producción de Genética Superior (CENPROGEN-SUP)» in Valle de Condebamba district, Cajamarca region, Peru, with the objective of selecting male guinea pigs for breeding based on their selection index. A

\footnotetext{
${ }^{1}$ Universidad Nacional Toribio Rodríguez de Mendoza de Amazonas, Amazonas, Perú

${ }^{2}$ Universidad Nacional de Cajamarca, Perú

${ }^{3}$ E-mail: saucedouriarte@gmail.com
}

Recibido: 23 de octubre de 2017

Aceptado para publicación; 9 de febrero de 2018 
total of 80 male guinea pigs of the Cajamarca ecotype of the Facultad de Ingeniería en Ciencias Pecuarias (pure and crossbred FICP) of the National University of Cajamarca, as well from the Valley of Mangallana and from the Valley of Cholocal. The guinea pigs were fed alfalfa, concentrate and water ad libitum for 56 days. The daily weight gain (IPD) and total weight gain (IPT), final weight (PF), body length (LC) and feed conversion (CA) were evaluated. The crossed FICP had significantly better values for IPD, IPT, PF and LC $(17.6 \pm 1.8 \mathrm{~g}, 979 \pm 98 \mathrm{~g}, 1328 \pm 134 \mathrm{~g}$ and $36.6 \pm 1.3 \mathrm{~cm}$, respectively) $(\mathrm{p}<0.05)$. On the other hand, there were no differences between groups for feed conversion. A high and significant correlation was found $(\mathrm{p}<0.05)$ between PF with LC and feed intake, and inverse correlation $(\mathrm{p}<0.05)$ between IPT and CA. The maximum value of the selection index corresponds to crossbred FICP guinea pigs $(0.873)$, followed by pure FICP $(0.544)$, Valley of Mangallana (0.400) and Valley of Cholocal (0.346), and $45 \%$ of the first quartile corresponds to crossbred FICP. Best germplasm with genetic superiority was found in the crossbred FICP population.

Key words: guinea pig; productive parameters; genetic merit; Cajamarca ecotype; selection index

\section{INTRODUCCIÓN}

El progreso genético mediante índices de selección beneficia la explotación intensiva de especies domésticas como el cuy. En el Perú se están realizando estudios en mejoramiento genético de esta especie enfocados en la estimación de parámetros genéticos, que influyen en las características productivas y reproductivas de su descendencia (Oblitas et al., 2006), los cuales son de gran importancia en la elaboración de herramientas como los índices reproductivos más altos para la selección de reproductores (Solarte y Viteri, 2001). El índice de selección es una herramienta apropiada para elegir los mejores ejemplares desde un punto de vista genético y económico (Henao, 1994), ya que se considera un valor diferenciado para cada carácter, según la conveniencia del productor. Para su elaboración, se debe determinar por separado el valor para cada carácter a seleccionar, y la sumatoria brindará el índice total (Vergara e Iriarte, 2002). Esta prueba permite la selección genética para poder cruzar los reproductores superiores a fin de incrementar la productividad de las características de interés para los productores (Chauca, 1997; Calvache, 2015).

Según Chauca (2007), varios programas del Instituto Nacional de Innovación Agraria (INIA) han avanzado, principalmente en mejoramiento genético de cuyes, pero conservando el germoplasma de los ecotipos locales de Lima, Cajamarca, Junín, Ayacucho, Cusco y Puno. En la Facultad de Ingeniería en Ciencias Pecuarias de la Universidad Nacional de Cajamarca (UNC), Perú, se viene trabajandoen el mejoramiento genético de cuyes del ecotipo Cajamarca con el fin de mejorar sus índices productivos $\mathrm{y}$ reproductivos. Así mismo, en el valle de Condebamba, Cajamarca, existen asociaciones de productores de cuyes (Aprocuyco, Coprodescuy), que constituyen las principales abastecedoras de la provincia (INEI, 2012); sin embargo, existe la necesidad de mejorar sus parámetros productivos mediante la introducción de reproductores en los planteles locales. 
El objetivo de la presente investigación fue determinar el mérito genético de cuyes machos del ecotipo Cajamarca, en función a sus parámetros productivos.

\section{Materiales y Métodos}

\section{Ubicación del Estudio}

El estudio se realizó entre mayo y junio de 2015 en la granja de cuyes «CENPROGEN-SUP», localizado en el distrito de Valle de Condebamba, región Cajamarca, a $2024 \mathrm{msnm}$, humedad relativa de $70-80 \%$ y temperatura ambiental anual de $16-18{ }^{\circ} \mathrm{C}$. La fase de campo duró 56 días pos-adaptación.

\section{Grupo Experimental}

Se evaluaron 80 cuyes machos del ecotipo Cajamarca, de 15 días de edad al destete (Quintana et al., 2013), con 15 días de adaptación y peso promedio de $312 \mathrm{~g}$ a los 30 días de edad. Los animales procedieron de la Facultad de Ingeniería en Ciencias Pecuarias de la UNC (FICP cruzados [A], FICP puros [B]), Valle de Mangallana (C, cuatro granjas) y Valle de Cholocal (D, seis granjas), con 20 individuos en cada grupo. Los FICP cruzados fueron hijos en la F1 de FICP puros con cuyes del ecotipo Cajamarca procedentes del distrito de Jesús.

\section{Sistema de Crianza}

Los cuyes fueron alimentados con forraje verde de alfalfa y un concentrado con la siguiente composición nutricional: materia seca $86 \%$, proteína cruda $18 \%$, energía digestible $3000 \mathrm{kcal} / \mathrm{kg}$, fibra $8 \%$, calcio $1 \%$, fosforo $0.5 \%$, lisina $0.9 \%$, metionina $0.6 \%$ y triptófano $0.25 \%$, así como suministro diario de agua ad libitum. Los cuyes se mantuvieron en jaulas individuales de 45x50x45 cm durante 71 días, donde 15 días fueron de adaptación a los alimentos ad libitum. La toma de datos duró 56 días pos-adaptación.
Los cuyes fueron tratados contra parásitos internos y externos con Biomisil ${ }^{\circledR} 0.1 \%$ (Ivermectina $0.1 \%$, ) administrado por vía subcutánea en dosis de $1 \mathrm{ml}$ por $5 \mathrm{~kg}$ de peso vivo. Además, se aplicó dosis vitamínica de solución de Complejo B Electrolitos ${ }^{\circledR}$ por vía oral, en dosis de una cucharadita por animal. Evaluación de Parámetros Productivos

Los cuyes fueron pesados al inicio (30 días de edad) y al final (PF) del estudio en una balanza digital con capacidad máxima de $5 \mathrm{~kg}$ y $0.01 \mathrm{~g}$ de precisión. El incremento de peso total (IPT) se determinó mediante la diferencia del peso final e inicial. El incremento de peso diario (IPD) fue el IPT dividido entre el número de días evaluados. La longitud corporal (LC) fue medida con regla milimetrada desde el extremo del hocico hasta el nivel del corvejón, registrándose el promedio de tres repeticiones por cuy. La conversión alimenticia (CA) se calculó dividiendo la cantidad de alimento consumido (alimento suministrado - alimento rechazado) entre el incremento de peso.

\section{Evaluación del Mérito Genético}

Para la obtención del índice de selección se consideró el IPD, PF, LC y CA con valores económicos de $0.3\left(\mathrm{~V}_{1}\right), 0.3\left(\mathrm{~V}_{2}\right), 0.2$ $\left(\mathrm{V}_{3}\right)$ y $0.2\left(\mathrm{~V}_{4}\right)$ respectivamente, que fueron asignados de acuerdo con el interés de la granja. Se usaron valores de heredabilidad $\left(\mathrm{h}^{2}\right)$ de estudios previos en la granja de cuyes «CENPROGEN-SUP»: IPD (0.4), PF (0.45), LC (0.45) y CA (0.4) (J. Mantilla, Cajamarca, comunicación personal). P representa el valor individual en función a su distribución normal, mediante la fórmula: $\mathrm{Z}=$ (media individual - media poblacional) / desviación estándar.

Los datos se aplicaron en la siguiente fórmula: IS $=\mathrm{V}_{1} \mathrm{~h}^{2}{ }_{1} \mathrm{P}_{1}+\mathrm{V}_{2} \mathrm{~h}_{2}{ }_{2} \mathrm{P}_{2}+\mathrm{V}_{3} \mathrm{~h}_{3}{ }_{3} \mathrm{P}_{3}$ - $\mathrm{V}_{4} \mathrm{~h}_{4}{ }_{4} \mathrm{P}_{4}$, donde IS = Valor de índice de selección; $\mathrm{V}=$ valor económico asignado; $\mathrm{h}^{2}$ $=$ heredabilidad del carácter; $\mathrm{P}=$ Valor de distribución normal 
Cuadro 1. Parámetros productivos de cuyes macho del ecotipo Cajamarca de cuatro procedencias, evaluados a los 56 días del destete (Perú, 2015)

\begin{tabular}{lcccc}
\hline & $\begin{array}{c}\text { FICP } \\
\text { cruzados }\end{array}$ & FICP $^{1}$ puros & $\begin{array}{c}\text { Valle de } \\
\text { Cholocal }\end{array}$ & $\begin{array}{c}\text { Valle de } \\
\text { Mangallana }\end{array}$ \\
\hline $\begin{array}{l}\text { Número de cuyes } \\
\text { Peso inicial }(\mathrm{g})^{2}\end{array}$ & 20 & 20 & 20 & 20 \\
$\begin{array}{l}\text { Incremento de peso } \\
\text { diario (g/día) }\end{array}$ & $17.6 \pm 1.8^{\mathrm{a}}$ & $16.4 \pm 1.6^{\mathrm{ab}}$ & $15.4 \pm 2.1^{\mathrm{b}}$ & $15.2 \pm 2.8^{\mathrm{b}}$ \\
$\begin{array}{l}\text { Incremento de peso } \\
\text { total (g) }\end{array}$ & $979 \pm 98^{\mathrm{a}}$ & $919 \pm 92^{\mathrm{ab}}$ & $865 \pm 150^{\mathrm{b}}$ & $853 \pm 156^{\mathrm{b}}$ \\
$\begin{array}{l}\text { Peso final (g) } \\
\begin{array}{l}\text { Longitud corporal } \\
\text { (cm) }\end{array}\end{array}$ & $1329 \pm 134^{\mathrm{a}}$ & $1208 \pm 107^{\mathrm{b}}$ & $1167 \pm 151^{\mathrm{b}}$ & $1159 \pm 169^{\mathrm{b}}$ \\
$\begin{array}{l}\text { Consumo de } \\
\text { materia seca }(\mathrm{g})\end{array}$ & $3508 \pm 1.3^{\mathrm{a}}$ & $36.0 \pm 1.3^{\mathrm{a}}$ & $35.6 \pm 1.4^{\mathrm{a}}$ & $34.3 \pm 1.8^{\mathrm{b}}$ \\
$\begin{array}{l}\text { Conversión } \\
\text { alimenticia }\end{array}$ & $4.1 \pm 0.6^{\mathrm{a}}$ & $4398 \pm 179^{\mathrm{ab}}$ & $3354 \pm 143^{\mathrm{b}}$ & $3297 \pm 235^{\mathrm{b}}$ \\
\hline
\end{tabular}

$a, b, c$ Letras diferentes dentro de filas representan diferencias significativas $(p<0.05)$

${ }^{1}$ FICP: Facultad de Ingeniería en Ciencias Pecuarias, Universidad Nacional de Cajamarca

${ }^{2} \mathrm{~A}$ los 30 días de edad

\section{Análisis Estadístico}

Se analizó la homogeneidad y normalidad con la prueba de Levene y Shapiro-Wilk respectivamente. Los datos fueron evaluados mediante un diseño completamente aleatorizado utilizando el análisis de varianza y la prueba de Tukey $(\mathrm{p}=0.05)$ para las comparaciones múltiples. Para evaluar la correlación de variables se empleó la prueba de Pearson $(\mathrm{p}=0.05)$. Los análisis se realizaron en la versión de prueba del software SPSS v 15.0 .

\section{Resultados}

Se hallaron diferencias al nivel $\mathrm{p}<0.05$ para peso inicial y peso final de los cuyes del grupo A respecto a B, C y D. No se encon- tró diferencias significativas entre los fenotipos FICP (A, B), así como tampoco entre los fenotipos de los valles $(\mathrm{C}, \mathrm{D})$ para IPD e IPT; sin embargo, los valores fueron mayores en cuyes de A que en cuyes de $\mathrm{C} y$ $\mathrm{D}(\mathrm{p}<0.05)$. Los cuyes de los grupos A, B y $\mathrm{C}$ tuvieron una mayor longitud corporal que aquellos del grupo $\mathrm{D}(\mathrm{p}<0.05)$. Asimismo, los cuyes del grupo A presentaron un mayor consumo de materia seca que los cuyes de los grupos $\mathrm{C}$ y $\mathrm{D}(\mathrm{p}<0.05)$. Por último, no hubo diferencias significativas entre grupos con relación a CA (Cuadro 1).

Se encontró una correlación alta entre el peso final y la longitud corporal $(\mathrm{r}=0.84$; $\mathrm{p}<0.05$ ), así como entre el consumo de alimento y el peso final $(r=0.71 ; p<0.05)$. Existe correlación inversa y media $(\mathrm{r}=-0.70, \mathrm{p}<0.05)$ entre el incremento de peso total y CA (Figura 1). 


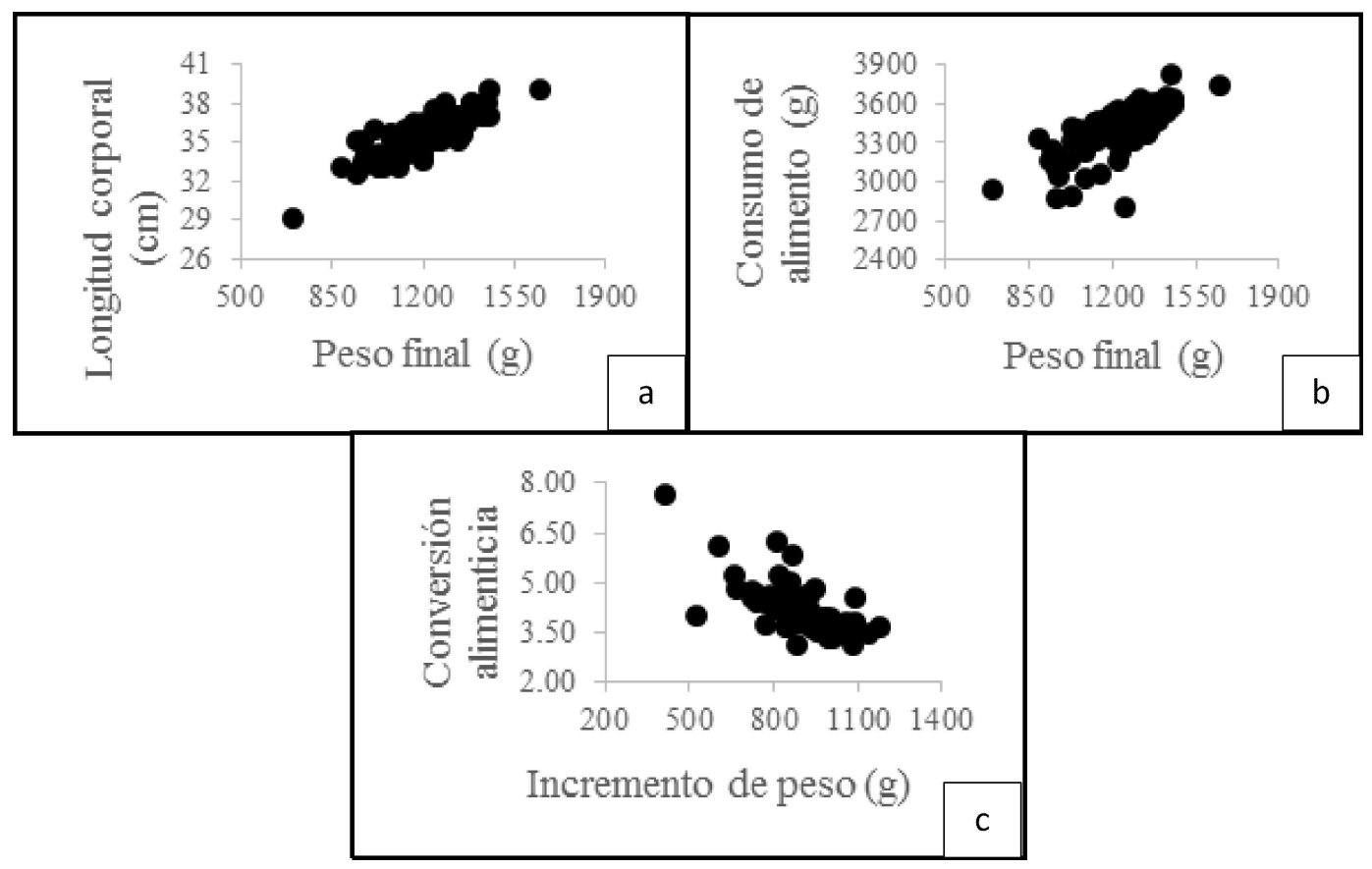

Figura 1. Correlación de parámetros productivos de cuyes macho del ecotipo Cajamarca a los 56 días pos-adaptación para la evaluación del mérito genético. (a) Peso final vs. longitud corporal. (b) Peso final vs. consumo de alimento. (c) Incremento de peso vs. conversión alimenticia

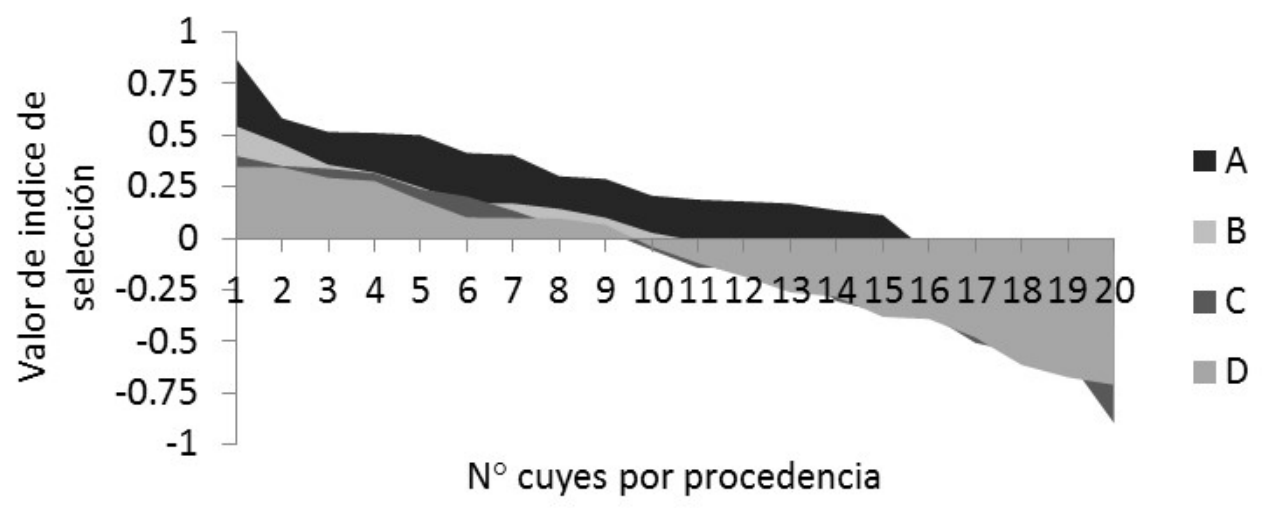

Figura 2. Distribución del mérito genético por índices de selección de cuyes machos del ecotipo Cajamarca de cuatro procedencias: FICP cruzados (A), FICP puros (B), Valle de Mangallana (C) y Valle de Cholocal (D) en función a parámetros productivos

Los cuyes A mostraron los mejores índices de selección con valor máximo de 0.873 , $\mathrm{B}$ con $0.544, \mathrm{C}$ con 0.400 y D con 0.346 (Figura 2). El $45 \%$ del primer cuartil de la población total corresponde a cuyes del grupo A, mientras que B y C aportan con el $20 \%$ y el $15 \%$ restante proviene del grupo D.

\section{Discusión}

Los IPD son superiores a los obtenidos por Camino e Hidalgo (2014) quiénes reportaron una media de $13.6 \mathrm{~g}$ /día en cuyes del genotipo Perú hasta las nueve semanas 
posdestete, debido posiblemente a la composición del alimento y a factores geográficos y genéticos (Sarria, 2011). Además, son superiores al reporte de Meza (2014) de 7.31 g/día, posiblemente debido a que en dicho estudio los cuyes fueron alimentados con gramíneas y plantas forrajeras arbustivas tropicales.

El IPT y el PF fueron similares a los resultados hallados por Camino e Hidalgo (2014), con 937 y $1237 \mathrm{~g}$, respectivamente, hasta las 12 semanas en cuyes de genotipos Perú y Cieneguilla. No obstante, es posible que los valores del presente estudio serían superiores si se hubieran seguido los animales hasta las 12 semanas. Son superiores a los reportes de Carvajal y Vivas (2008) en cuyes tipo 1 de la línea Perú, así como a los valores reportados por Bazay et al. (2014) en cuyes de la línea Inti hasta la sexta semana de edad y superiores en consumo de materia seca. En general, el genotipo tiene influencia en la ganancia de peso, ya que puede estar relacionado con el grado de heterosis de las poblaciones (Lasley, 1991), como lo cuyes del ecotipo Cajamarca de este estudio. Además, estos parámetros podrían verse afectados por los factores geográficos (altitud, latitud, etc.) y nutricionales (Camino e Hidalgo, 2014).

Morales et al. (2011) y Camino e Hidalgo (2014) reportaron conversiones de 3.23 y 3.54 en cuyes del genotipo Perú, siendo superiores al de este estudio, posiblemente debido a que el genotipo Perú está especializado en la eficiencia del consumo de alimento, a diferencia del ecotipo Cajamarca que está en proceso de mejoramiento genético (Chauca, 2007). Mantilla (2012) halló una CA de 4.32 en cuyes del ecotipo Cajamarca, siendo similar a los hallazgos de este estudio, así como Carballo (2015) con 4.74 en cuyes peruanos mejorados. Sin embargo, Mattos et al. (2003) reportaron valores de conversión menores (6.1) en cuyes de ambos sexos de la línea Perú.

Díaz (2012) encontró un LC de 37, 34 y $37 \mathrm{~cm}$ en cuyes de la línea Perú, Andina e
Inti, respectivamente, a los 3 meses de edad, siendo similares a los del presente estudio. Esta similitud podría deberse a que la línea genética podría tener bajo efecto sobre LC de cuyes mejorados. No obstante, los resultados de este estudio fueron superiores al reporte de Carballo (2015), con $30.5 \mathrm{~cm}$ medidos hasta la última vertebra sacra. Por otro lado, Enríquez (1978) halló una correlación alta $(r=0.71)$ entre PF y LC, similar a este estudio.

\section{Conclusiones}

- El uso de los índices de selección permite la identificación de los cuyes con aptitud productiva que pueden ser utilizados en un plan de mejoramiento genético.

- El mayor mérito genético corresponde a la población de cuyes FICP cruzados, pues fueron superiores en incremento de peso diario y total y peso final.

\section{Literatura Citada}

1. Bazay G, Carcelén F, Ara M, Jiménez R, Gonzales R, Quevedo W. 2014. Efecto de los manano-oligosacaridos sobre los parámetros productivos de cuyes (Cavia porcellus) durante la fase de engorde. Rev Inv Vet Perú 25: 198204. doi: 10.15381/rivep.v25i2.8491

2. Calvache CE. 2015. Evaluación genética de cuyes (Cavia porcellus) en Nariño - Colombia mediante inferencia Bayesiana y frecuentista. Tesis de Ingeniero Zootecnista. San Juan de Pasto, Colombia: Univ. de Nariño. 84 p.

3. Camino J, Hidalgo V. 2014. Evaluación de dos genotipos de cuyes (Cavia porcellus) alimentados con concentrado y exclusión de forraje verde. Rev Inv Vet Perú 25: 190-197. doi: 10.15381/ rivep.v25i2.8490

4. Carbajal J, Vivas N. 2008. Evaluación del remplazo parcial del forraje Axonopus sp por Saccharina rustica en 
la alimentación del cuy (Cavia porcellus). Rev Cub Cien Agric 42: 275-277.

5. Carballo L. 2015. Evaluación del crecimiento y engorde mediante la implantación de tres dosis de zeranol $(0.5 \mathrm{mg}, 1 \mathrm{mg}, 1.5$ $\mathrm{mg}$ ) en cuyes peruanos mejorados, Guaranda, Provincia Bolivar. Tesis de Médico Veterinario y Zootec-nista. Ecuador: Univ. Estatal de Bolivar. 121 p.

6. Chauca L. 1997. Producción de cuyes (Cavia porcellus). Roma: Organización de las Naciones Unidas para la Agricultura y la Alimentación. 120 p.

7. Chauca L. 2007. Logros obtenidos en la mejora genética del cuy (Cavia porcellus) experiencias del INIA. Arch Latinoam Prod Anim 15: 218-222.

8. Díaz M. 2012. Estudio filogenético de tres líneas de cuyes (Cavia porcellus L), Perú, Andina e Inti en la hacienda «E1 Prado». Tesis de Ingeniero Agropecuario. Sangolquí, Ecuador: Escuela Politécnica del Ejército. $106 \mathrm{p}$.

9. Enríquez M. 1978. Estimación de algunos parámetros biológicos y su interrelación con el peso vivo y número de crías por camada en cobayos. Tesis Doctoral. Lima, Perú: Univ. Nacional Agraria La Molina. $46 \mathrm{p}$.

10. Henao F. 1994. Principios de genética y mejoramiento animal. Santa Fe de Bogotá: UNISUR. 372 p.

11. [INEI] Instituto Nacional de Estadística e Informática. 2012. IV Censo Nacional Agropecuario. [Internet]. Disponible en: http://censos.inei.gob.pe/ cenagro/tabulados/

12. Lasley J. 1991. Genética del mejoramiento del ganado. $2^{\mathrm{a}}$ ed. México: Ed Limusa. $378 \mathrm{p}$.

13. Mantilla J. 2012. Diferenciación reproductiva, productiva y molecular de cuyes nativos de la región Cajamarca. Tesis Doctoral. Cajamarca, Perú: Univ. Nacional de Cajamarca. 149 p.
14. Mattos C, Chauca L, San Martín H, Carcelén C, Arbaiza F. 2003. Uso del ensilado biológico de pescado en la alimentación de cuyes mejorados. Rev Inv Vet Perú 14: 89-96. doi: 10.15381/ rivep.v14i2.1612

15. Meza A, Cabrera R, Morán J, Meza F, Cabrera C, Meza C, Meza J, et al. 2014. Mejora de engorde de cuyes ( $\mathrm{Ca}$ via porcellus $\mathrm{L}$ ) a base de gramíneas y forrajeras arbustivas tropicales en la zona de Quevedo, Ecuador. Idesia (Arica) 32: 75-80. doi: 10.4067/S0718-34292014000300010

16. Morales M, Carnelén C, Ara G, Arbaiza F, Chauca F. 2011. Evaluación de dos niveles de energía en el comportamiento productivo de cuyes (Cavia porcellus) de la raza Perú. Rev Inv Vet Perú 22: 177-182. doi: 10.15381/ rivep.v22i3.254

17. Oblitas J, Oscanoa W, Kajjak N. 2006. Parámetros genéticos en cuyes de la Estación Experimental INIA Huancayo. En: XXIX Reunión APPA. Huancayo, Perú: Asociación Peruana de Producción Animal.

18. Quintana E, Jiménez R, Carcelén F, San Martín H, Ara M. 2013. Efecto de dietas de alfalfa verde, harina de cebada y bloque mineral sobre la eficiencia productiva de cuyes. Rev Inv Vet 24: 425-432. doi: 10.15381/rivep.v24i4.2744

19. Solarte C, Viteri L. 2001. Índice de selección prueba de progenie, y prueba de comportamiento en cuyes. Rev Zootec 4: 35-44

20. Sarria BJ. 2011. El cuy. Crianza tecnificada. Manual técnico encuyicultura N. ${ }^{\circ}$ 1. Lima: Univ. Nacional Agraria La Molina. $64 \mathrm{p}$.

21. Vergara O, Iriarte C. 2002. Determinación de un Índice de selección para el peso al nacer y al destete en ganado Cebú. MVZ Córdoba 7: 148-151. 